on $\mathrm{CE}$. The demand for $\mathrm{DBE}$ is rising and it is fast becoming a necessity in the investigation algorithm of the small bowel.

Competing interests None.

Keywords capsule endoscopy, double balloon enteroscopy.

\section{PTH-136 DOUBLE BALLOON ENTEROSCOPY IN THE ERA OF CAPSULE ENDOSCOPY: NECESSITY OR LUXURY?}

doi:10.1136/gut.2011.239301.537

R Sidhu, 1,* M E McAlindon, ${ }^{1}$ S Hardcastle, ${ }^{1}$ D S Sanders ${ }^{1}$ 'Gastroenterology, Royal Hallamshire Hospital, Sheffield, UK

Introduction The use of double balloon enteroscopy (DBE) in the UK has been relatively slow and limited to tertiary centres. The authors evaluated the demand for DBE in the UK and impact on patient management.

Methods Data was collected prospectively for all DBE procedures performed since 2006. All patients underwent CE (either at our centre or elsewhere) prior to DBE. The majority of $\mathrm{CE}$ was positive which directed the route for DBE. Patient demographics, indications, procedural details and change in management were collected.

Results A total of 166 procedures were carried out in 121 patients over 50 months (85 oral route, 81 retrograde route). $74 \%$ of the referrals were from outside the region. There were 65 females with a median age of 52 years (range $20-83$ years). The most common indication for DBE was iron deficiency anaemia (IDA) in 43\% followed by Crohn's disease in $32 \%$ and overt bleeding in $12 \%$. The median procedure time was 66 min (2-160 min). Pan enteroscopy was achieved in one patient. The overall diagnostic yield for DBE was $46 \%$ for all indications. The complication rate was $0.8 \%$ with one case of pancreatitis postpolypectomy which was managed conservatively. Four patients with small bowel tumours on CE were referred for surgery as the tumour was not reached at DBE. The yield for DBE for vascular lesions was $52 \%$ $(15 / 29)$. In two patients from this cohort, alternative diagnosis were found while a repeat CE locally was normal in 3 other patients. Polyps were seen in 6 patients on CE, 4 of these underwent polypectomy at DBE. In the 2 remaining patients, the isolated polyp at CE was thought to be incidental and not pursued. Therapeutic intervention was performed during DBE in $20 \%$ ( $n=24$ : argon plasma coagulation $(n=17)$, endoscopic mucosal resection $(n=5)$ and endo clips $(n=2)$. Patient management was altered in $38 \%$ of the cohort after DBE which included intervention at DBE $(n=24)$, referral for surgery $(n=2)$, inflammatory bowel disease directed therapy $(n=6)$, helicobacter pylori eradication $(n=1)$, chemotherapy $(n=1)$ and stopping NSAIDs $(n=1)$. The technique of DBE is associated with a learning curve. In this series, the diagnostic yield in the latter 50 patients was $56 \%$ compared to $40 \%$ in the initial 50 patients $(p=0.1)$. The procedural time also decreased but was not statistically significant (70 vs 63 min, $p=0.1$ ). In 2009, for every 17 CE's performed one patient underwent DBE locally.

Conclusion DBE has a high diagnostic yield, low complication rate and positive impact on patient management with the ability to perform biopsies or therapeutics for pathology seen 